\title{
Lepton flavor violation induced by a neutral scalar at future lepton colliders
}

\section{P. S. Bhupal Dev}

Department of Physics and McDonnell Center for the Space Sciences, Washington University, St. Louis, MO 63130, USA

E-mail: bdevewust 1. edu

\section{Rabindra N. Mohapatra}

Maryland Center for Fundamental Physics, Department of Physics, University of Maryland, College Park, MD 20742, USA

E-mail: rmohapat@umd.edu

\section{Yongchao Zhang*}

Department of Physics and McDonnell Center for the Space Sciences, Washington University, St. Louis, MO 63130, USA

E-mail: yongchao.zhang@physics.wustl.edu

\begin{abstract}
Many new physics scenarios beyond the Standard Model often necessitate the existence of a neutral scalar $\mathrm{H}$, which might couple to the charged leptons in a flavor violating way, while evading all existing constraints. Such scalars could be effectively produced at future lepton colliders like CEPC, ILC and FCC-ee, either on-shell or off-shell, and induce lepton flavor violating (LFV) signals. We find that a large parameter space of the scalar mass and the LFV couplings can be probed, well beyond the current low-energy constraints in the lepton sector. In particular, a scalarloop induced explanation of the longstanding muon $g-2$ anomaly can be directly tested in the on-shell mode.
\end{abstract}

ICHEP 2018, XXXIX International Conference on High Energy Physics

4-11 July 2018

COEX, Seoul, Korea

${ }^{*}$ Speaker. 


\section{Introduction}

There are various theoretical models of new physics which lead to charged lepton flavor violation (cLFV) effects at an observable level. They generally involve extending the Higgs sector, which allows flavor-violating Yukawa couplings of new neutral scalars beyond the standard model (SM). Some well-motivated examples include supersymmetric models with leptonic $R$-parity violation, left-right symmetric models, mirror models, and two-Higgs doublet models, where the cLFV couplings might arise at tree or loop level.

In this paper, we show that such scenarios of neutral scalar-induced cLFV can be effectively probed in a model-independent way at future lepton colliders, such as the CEPC, ILC, FCC-ee and CLIC. Here we include both on and off-shell production of the new neutral scalar $H$ at lepton colliders, which enables us to derive the lepton flavor violation (LFV) sensitivity as a function of the mass $m_{H}$ for a direct comparison with the current bounds from low-energy experiments. Moreover, for $m_{H}$ small compared to the center-of-mass energy, the effective four-fermion approximation does not work. Without loss of generality, we can write the effective Yukawa couplings of $H$ to the charged leptons as

$$
\mathscr{L}_{Y}=h_{\alpha \beta} \bar{\ell}_{\alpha, L} H \ell_{\beta, R}+\text { H.c. . }
$$

Here for simplicity we assume the couplings $h_{\alpha \beta}$ are all real and chirality-independent and thus symmetric. We assume futher that $H$ is $\mathrm{CP}$ even and its mixing with and/or coupling to the SM Higgs is small. With an $\mathrm{ab}^{-1}$ level of integrated luminosity, a large parameter space of $m_{H}$ and $h_{\alpha \beta}$ could be probed, well beyond the current cLFV constraints. More details can be found in Ref. [1], and the application to the left-right symmetric models is given in Ref. [2].

\section{On-shell LFV}

If kinematically allowed, the neutral scalar $H$ can be directly produced at lepton colliders, in association with a pair of flavor-changing leptons through the couplings in Eq. (1.1), i.e. $e^{+} e^{-} \rightarrow$ $\ell_{\alpha}^{ \pm} \ell_{\beta}^{\mp} H$ (with $\alpha \neq \beta$ ). Let us first switch on the coupling $h_{e \mu}$ and off all other (LFV) couplings. It should be emphasized that the production amplitudes depend only on the LFV couplings $h_{\alpha \beta}$ (here $\alpha \beta=e \mu$ ), and thus could be easily made to satisfy the rare lepton decay constraints, such as $\mu \rightarrow e e e$ and $\mu \rightarrow e \gamma$, which depend on the product $\left|h_{e e}^{\dagger} h_{e \mu}\right|$. We have only the following constraints: (i) Muonium-antimuonium oscillation: This could occur in both $s$ and $t$-channels, with the oscillation probability $\mathscr{P} \propto\left|h_{e \mu}\right|^{4} / m_{H}^{4}$. (ii) The anomalous magnetic moment of electron $a_{e}$ receives a contribution from the $H-\mu$ loop. (iii) the LEP $e^{+} e^{-} \rightarrow \mu^{+} \mu^{-}$data [3]: A $t$-channel $H$ could mediate the scattering $e^{+} e^{-} \rightarrow \mu^{+} \mu^{-}$, which interferes with the SM diagrams in the $s-$ channel. All these constraints on $m_{H}$ and $h_{e \mu}$ are shown in the left panel of Fig. 1 as the shaded regions. To explain the longstanding theoretical and experimental discrepancy of the muon $g-2$, i.e. $\Delta a_{\mu}=(2.87 \pm 0.80) \times 10^{-9}$ [4], the LFV coupling $h_{e \mu}$ is required to be larger, which is already excluded by the $(g-2)_{e}$ data.

To be specific, we consider two benchmark configurations for future lepton colliders: i.e. the CEPC $240 \mathrm{GeV}$ with an integrated luminosity of $5 \mathrm{ab}^{-1}$ and ILC $1 \mathrm{TeV}$ witha a luminosity of 1 $\mathrm{ab}^{-1}$. The SM background is dominated by particle mis-identification, e.g. $e$ mis-identified as $\mu$ 

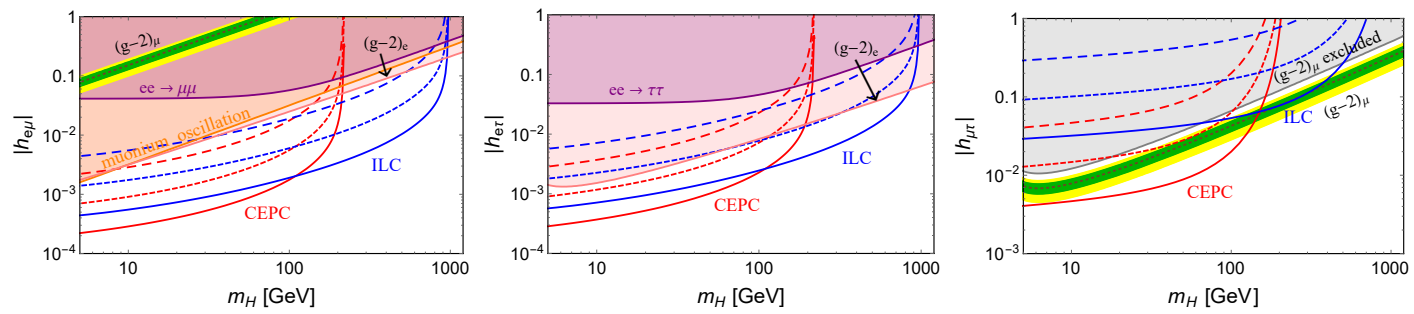

Figure 1: Prospects of probing LFV couplings $h_{\alpha \beta}(\alpha \neq \beta)$ from searches of $e^{+} e^{-} \rightarrow \ell_{\alpha}^{ \pm} \ell_{\beta}^{\mp} H$ at CEPC $240 \mathrm{GeV}$ (red) and ILC $1 \mathrm{TeV}$ (blue). Here we have assumed $10 \mathrm{LFV}$ signal events and a BR of $1 \%$ (longdashed) $10 \%$ (short-dashed) or 100\% (solid) from $H$ decay to be visible. In the plots all the shaded regions are excluded by the constraints as labelled. In the left and right panels, the brown line could fit the central value of $\Delta a_{\mu}$, and the green and yellow bands cover the $1 \sigma$ and $2 \sigma$ ranges of $\Delta a_{\mu}$.
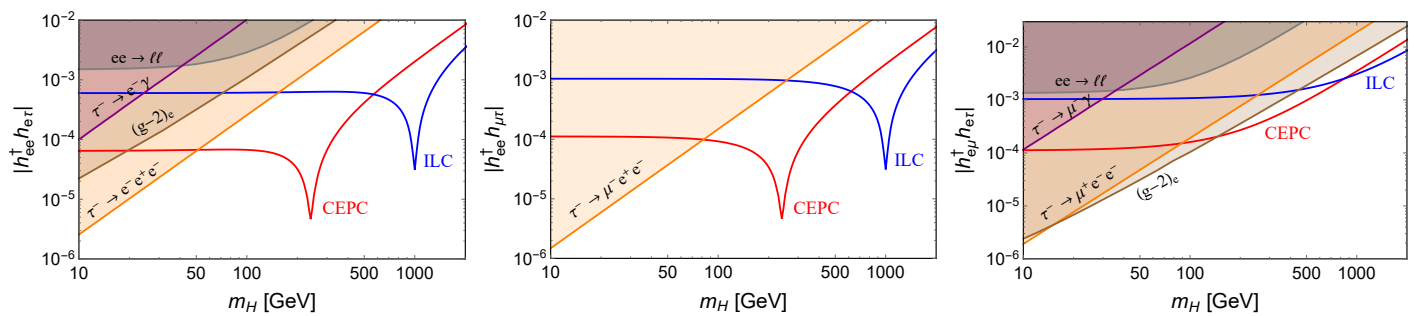

Figure 2: Prospects of $\left|h_{e e}^{\dagger} h_{e \tau}\right|$ (left), $\left|h_{e e}^{\dagger} h_{\mu \tau}\right|$ (middle) and $\left|h_{e \mu}^{\dagger} h_{e \tau}\right|$ (right) from searches of $e^{+} e^{-} \rightarrow$ $e^{ \pm} \tau^{\mp}, \mu^{ \pm} \tau^{\mp}$ at CEPC $240 \mathrm{GeV}$ (red) and ILC $1 \mathrm{TeV}$ (blue). Here we have assumed 10 signal events. Also shown are the constraints from the rare lepton decays, $(g-2)_{e}$, and the LEP $e^{+} e^{-} \rightarrow \ell^{+} \ell^{-}$data [3].

or vice versa, and is very small. After being produced, $H$ could decay back into the charged lepton pairs or other SM particles. To work in a model-independent way, we consider three benchmark values, where $1 \%, 10 \%$ or $100 \%$ of the decay products of $H$ are visible and can be reconstructed. The corresponding LFV prospects are shown in the left panel of Fig. 1, where we have assumed a minimum of 10 signal events at both CEPC and ILC. It is clear from Fig. 1 that with a branching ratio (BR) of $\gtrsim 10 \%$, a large region of $m_{H}$ and $\left|h_{e \mu}\right|$ can be probed in future lepton colliders, which extends the limits well beyond what is currently available.

Turning now to the coupling $h_{e \tau}$, the most stringent limit comes from the electron $g-2$, and the LEP $e^{+} e^{-} \rightarrow \tau^{+} \tau^{-}$data [3]. The reconstruction of $\tau$ lepton is more challenging than $\mu$, but there is still ample parameter space to probe at both CEPC and ILC, as long as the effective BR is $\gtrsim 10 \%$. Regarding the coupling $h_{\mu \tau}$, there are currently no experimental limits, except for the muon $g-2$ discrepancy. This could be explained in presence of $H$ when it couples to muon and tau, as shown by the brown line and the green and yellow bands in the right panel of Fig. 1, while the shaded region is excluded by the current muon $g-2$ data at the $5 \sigma$ level.

\section{Off-shell LFV}

The LFV signals could also be produced from an off-shell $H$, i.e. $e^{+} e^{-} \rightarrow \ell_{\alpha}^{ \pm} \ell_{\beta}^{\mp}$. This could occur in both the $s$ and $t$ channels; in the $s$-channel $H$ is on-shell if the colliding energy $\sqrt{s} \simeq m_{H}$ (resonance). Different from the on-shell case, the off-shell production amplitudes have a quadratic dependence on the Yukawa couplings (some of them might be flavor conserving), and thus largely complementary to the on-shell LFV searches. 
The amplitude $e^{+} e^{-} \rightarrow e^{ \pm} \mu^{\mp}$ is proportional to $h_{e e}^{\dagger} h_{e \mu}$. This is tightly constrained by the $\mu \rightarrow$ eee data, leaving no hope to see any signal in this channel. In the $\tau$ lepton sector, the LFV decay constraints are comparatively much weaker. The limits on $\left|h^{\dagger} h\right| / m_{H}^{2}$ from $\tau^{-} \rightarrow e^{-} \gamma$ and $\tau^{-} \rightarrow e^{+} e^{-} e^{-}$are almost constants, as in effective field theories with superheavy mediators. As for the on-shell case above, the coupling $\left|h_{e e}^{\dagger} h_{e \tau}\right|$ are constrained by the electron $g-2$ and the LEP $e^{+} e^{-} \rightarrow \ell^{+} \ell^{-}$data [3]. All the constraints are presented in the left panel of Fig. 2.

The dominant SM backgrounds are from the process $e^{+} e^{-} \rightarrow W^{+} W^{-} \rightarrow e^{-} \tau^{+} \bar{v}_{e} v_{\tau}$ which is expected to be small. Assuming 10 signal events as above, the prospects of the coupling $\left|h_{e e}^{\dagger} h_{e \tau}\right|$ are shown in Fig. 2. At the resonance $m_{H} \simeq \sqrt{s}$, the production cross section can be greatly enhanced by $m_{H}^{2} / \Gamma_{H}^{2}$. To be specific, we have set the width $\Gamma_{H}=10$ (30) GeV at $\sqrt{s}=240 \mathrm{GeV}(1 \mathrm{TeV})$. For $m_{H}>\sqrt{s}$, the production rate diminishes rapidly as $H$ becomes heavier. An off-shell $H$ could however be probed up to a few-TeV range, as shown in Fig. 2, and ILC is expected to be more promising than CEPC in this mass range, as a result of the higher $\sqrt{s}$.

The process $e^{+} e^{-} \rightarrow \mu^{ \pm} \tau^{\mp}$ could proceed via both the $s$ and $t$ channels, which depend on different couplings, namely $\left|h_{e e}^{\dagger} h_{\mu \tau}\right|$ and $\left|h_{e \mu}^{\dagger} h_{e \tau}\right|$, and are constrained respectively by the rare decays $\tau^{-} \rightarrow \mu^{-} e^{+} e^{-}$and $\tau^{-} \rightarrow \mu^{+} e^{-} e^{-}$. Analogous to the $e \tau$ case above, a broad range of $m_{H}$ and $\left|h_{e e}^{\dagger} h_{\mu \tau}\right|$ could be probed in the $s$ channel, in particular in vicinity of the resonance, as shown by the middle panel of Fig. 2. In the $t$ channel, the cross sections are comparatively smaller, and the detectable regions are much narrower, as shown by the right panel of Fig. 2.

\section{Conclusion}

We have shown that a hadrophobic neutral scalar $H$, which is well-motivated in a large class of new physics scenarios, can be probed in an $e^{+} e^{-}$collider via its LFV couplings to the charged lepton sector. We present a model-independent analysis of how far the LFV coupling strengths and the scalar mass can be probed beyond the existing limits from the low-energy sector. In particular, we find that the full mass and coupling range of the scalar, that can explain the muon $g-2$ anomaly, can be tested in the future lepton colliders. This is largely complementary to the searches of LFV in the low-energy experiments and hadron colliders.

\section{Acknowledgments}

This work is supported by the U.S. Department of Energy under Grant No. DE-SC0017987.

\section{References}

[1] P. S. B. Dev, R. N. Mohapatra and Y. Zhang, Phys. Rev. Lett. 120, no. 22, 221804 (2018) [arXiv:1711.08430 [hep-ph]].

[2] P. S. Bhupal Dev, R. N. Mohapatra and Y. Zhang, Phys. Rev. D 98, no. 7, 075028 (2018) [arXiv:1803.11167 [hep-ph]].

[3] J. Abdallah et al. [DELPHI Collaboration], Eur. Phys. J. C 45, 589 (2006) [hep-ex/0512012].

[4] M. Tanabashi et al. [Particle Data Group], Phys. Rev. D 98, no. 3, 030001 (2018). 